\section{Pesquisa do nistagmo/vertigem de posição e avaliação eletronistagmográfica em um grupo de individuos portadores de diabetes Mellitus tipo I}

\author{
Lílian P. Scherer', Fga. Marilda B. Lobo ${ }^{2}$
}

\section{Resumo}

$\mathbf{I}$

ntrodução: De acordo com a Sociedade Brasileira de Diabetes, estima-se que existam no Brasil cerca de 5 milhões de pessoas com a patologia. No estado do Rio Grande do Sul há, provavelmente, 400.000 pacientes diabéticos23,27. Dentre as alterações que os indivíduos portadores de diabetes Mellitus podem apresentar, estão incluídos os distúrbios vestibulares. Objetivo: $O$ presente trabalho teve por objetivo colaborar nas pesquisas de melhoria na qualidade de vida do paciente diabético e realizar pesquisa do nistagmo/ vertigem de posição e eletronistagmografia, a fim de identificar possíveis alterações. Forma de estudo: Clínico prospectivo. Material e método: 12 indivíduos portadores de diabetes Mellitus tipo I, usuários regulares de insulina, com idades entre 12 e 27 anos e componentes da Associação Riograndense de Apoio aos Diabéticos (ARAD). Para verificar as respostas vestibulares nos indivíduos portadores de diabetes Mellitus tipo I, foram realizados anamnese específica, meatoscopia, timpanometria, pesquisa do nistagmo/ vertigem de posição e eletronistagmografia na amostra selecionada. Resultados: Verificou-se Síndrome Vestibular Periférica Irritativa em 75\% dos resultados alterados. Destes, 62,5\% se tratavam de sujeitos sem queixa otoneurológica. Conclusões: Observou-se que o efeito do diabetes tipo I, na função vestibular, deve ser avaliado como as demais complicações diabéticas usuais, mesmo em pacientes assintomáticos, pois, com o diagnóstico etiológico precoce, é possível o auxílio na prevenção das complicações desta patologia.

\author{
Search of the nystagmus/ \\ positional vertigo and \\ electronystagmographic \\ evaluation in a group of diabetics \\ Mellitus type I
}

Palavras-chave: diabetes Mellitus, eletronistagmografia, síndrome vestibular irritativa, vertigem, labirintologia.

Key words: diabetes Mellitus, electronystagmographic, irritable vestibular sindrome, vertigo, labyrintholgy.

\section{Summary}

\begin{abstract}
I
ntroduction: According to the Brazilian Society of Diabetes, there are 5 million diabetics in Brazil. In the state of Rio Grande do Sul only, there are, probably, 400.000 diabetics23,27. This group of people can present vestibular alterations. Aim: This article had the objective to contribute with the searches to help improve the quality of life of the diabetics and to search the nystagmus/ positional vertigo and electronystagmographic evaluation, and to identify possible alterations. Study design: Prospective clinical. Material and method: 12 Diabetics Type I, insulin dependents, between 12 and 27 years old, and members of Associação Riograndense de Apoio aos Diabéticos (ARAD). To observe the vestibular reactions, we did a specific interview, otoscopy, tympanometric, search of the Nystagmus/ Positional Vertigo and Electronystagmographic Evaluation in the selected sample. Results: We verified Periferic Irritable Vestibular Syndrome in $75 \%$ of the altered results. $62,5 \%$ in this group didn't present otoneurologyc symptom. Conclusions: We note that the effect of Diabetes Type I in the vestibular function may be taken into consideration like any other usual complications, since the same may occur in asymptomatic patients, and also because with the early etiologic diagnostic it is possible to help in the prevention of this disease's complications.
\end{abstract}

\footnotetext{
${ }^{1}$ Graduanda em fonoaudiologia pela Universidade Luterana do Brasil

${ }^{2}$ Mestranda em Distúrbios da Comunicação Humana pela Universidade Federal de Santa Maria - RS. Instituição: Universidade Luterana do Brasil - Canoas - RS Endereço para correspondência Lílian Pacheco Scherer

Tel.: (0xx51)3222.6663 ou (0xx51)982.95190 - E-mail: lilascherer@hotmail.com
}

O trabalho foi aprovado pela Comissão de Aconselhamento de Ética em Pesquisa do Curso de Fonoaudiologia da Universidade Luterana do Brasil, conforme a resolução 196 do CONEP, em 31 de julho de 2001 e apresentado como quesito parcial para obtenção de graduação em fonoaudiologia na Universidade Luterana do Brasil, em 17 de dezembro de 2001.

Artigo recebido em 17 de janeiro de 2002. Artigo aceito em 14 de março de 2002. 


\section{INTRODUÇÃO}

Diabetes Mellitus é uma desordem crônica determinada geneticamente que resulta da perturbação do metabolismo da glicose causada por deficiência absoluta ou relativa de insulina ${ }^{1,2}$.

A insulina é uma proteína produzida pelo pâncreas, responsável pelo aproveitamento e metabolização da glicose pelas células do organismo, que tem como finalidade gerar energia $^{3}$.

O Diabetes Tipo I geralmente se manifesta antes dos 15 anos de idade, sendo caracterizado por início rápido e presença dos sintomas clássicos ${ }^{4}$.

O Diabetes Tipo II surge, geralmente, após os 40 anos de idade. Os sintomas são menos pronunciados e podem permanecer desapercebidos por muito tempo ${ }^{3,4,5}$.

Hoje há aproximadamente 135 milhões de diabéticos no mundo. Estima-se que existam no Brasil cerca de 5 milhões de pessoas com a patologia. No estado do Rio Grande do Sul há, provavelmente, 400.000 indivíduos portadores da síndromes.

Avanços importantes no estabelecimento de critérios diagnósticos e no conhecimento de novas estratégias de tratamento têm sido relatados nos últimos anos. Entretanto, muitas destas informações não estão ainda suficientemente consolidadas, dificultando ao médico clínico, que atende pacientes diabéticos, tomar decisões fundamentadas nos preceitos modernos da terapêutica.

O metabolismo da glicose tem grande influência no funcionamento da orelha interna. Bittar e col. ${ }^{6}$, Ferreira Jr. e col. ${ }^{7}$ e Ramos e col. ${ }^{8}$ afirmam que a orelha interna destacase no corpo humano por sua intensa atividade metabólica. No entanto, a estrutura não possui reserva energética armazenada, o que faz com que pequenas variações de glicose no sangue influenciem no seu funcionamento, provocando alterações do equilíbrio. De acordo com Mor e col. ${ }^{9}$, o espaço existente entre os labirintos ósseo e membranoso, na orelha interna, é preenchido por perilinfa, rica em sódio, enquanto que, na parte interna do labirinto membranoso há endolinfa, com maior quantidade de potássio.

Para Ferreira Jr. e col. ${ }^{7}$, na alteração metabólica, há tendência de deslocamento de potássio da endolinfa para perilinfa e de sódio em sentido contrário. Esse mecanismo provocaria vertigem, zumbido, hipoacusia e plenitude auricular.

Para o funcionamento adequado da orelha interna, é necessário um equilíbrio no nível de insulina e aporte adequado de glicose. No Diabetes Mellitus, há glicose no sangue, mas a substância não é capaz de entrar na orelha interna pelo déficit de insulina ${ }^{7}$, o que explicaria o que afirmou Fangchao ${ }^{10}$, pois o autor diz que os diabéticos que usam insulina têm melhores limiares auditivos que os diabéticos que não usam, referindo que o uso da insulina limitaria a progressão da perda auditiva.
Perez e col. ${ }^{11}$ acreditam que há prejuízo funcional do órgão vestibular no estado metabólico diabético.

O trabalho teve por objetivo:

- Realizar Pesquisa do Nistagmo e Vertigem de Posição; e Eletronistagmografia em sujeitos portadores de Diabetes Mellitus Tipo I e

- Identificar as possíveis alterações observáveis.

\section{MATERIAL E MÉTODO}

Realizou-se um estudo observacional, de grupo, transversal, contemporâneo e descritivo. Foram participantes da amostra desta pesquisa 12 indivíduos componentes da Associação Riograndense de Apoio aos Diabéticos; usuários de insulina; e portadores de Diabetes Mellitus Tipo I. Não foram avaliados portadores de Diabetes Mellitus Tipo 2 a fim de excluir possíveis fatores associados, como idade e obesidade, estando em acordo com os estudos de Biurrun e col. ${ }^{12}$, sendo $58,3 \%$ do sexo masculino e $41,7 \%$ do sexo feminino.

A coleta de dados transcorreu no período de 10 de agosto a 5 de outubro de 2001. O grupo controle não foi utilizado, pois segundo Caovilla e col. ${ }^{13}$, as curvas glicêmica e insulinêmica são consideradas fundamentais para a confirmação de alteração por hiperinsulinemia e, portanto, necessárias para a exclusão de alterações metabólicas glicêmicas em indivíduos considerados sadios e sem sintomas.

Nenhum sujeito da amostra foi excluído da avaliação por apresentar alterações de orelha externa e orelha média como malformações, otites, rolha de cerúmen e perfuração timpânica.

O critério de inclusão deste estudo constitui-se de todos indivíduos disponíveis à participação, portadores de Diabetes Mellitus Tipo I, adolescentes e adultos até a faixa de 40 anos. Foram excluídos do trabalho indivíduos com idade superior a 40 anos, para evitar a presença de outras manifestações decorrentes da idade, como a presbivertigem e a presbiataxia (desequilíbrio do idoso). Estas podem ser ocasionadas por processos degenerativos do sistema vestibular periférico e central, reduzindo a habilidade de combinar sinais visuais, proprioceptivos e vestibulares e de modificar os reflexos adaptativos ${ }^{14}$. Foram também excluídos os indivíduos que se recusaram ao deslocamento até a Universidade Luterana do Brasil para a realização dos exames.

Para verificar as respostas vestibulares na amostra citada, realizou-se:

- Anamnese específica utilizada para nortear o examinador ${ }^{15}$ e excluir indivíduos portadores de patologias que pudessem interferir nos resultados das provas, sem, no entanto, fazer parte do conjunto de análise de respostas.

- Meatoscopia: para viabilizar a colocação da sonda imitanciométrica e verificar a presença de malformações, rolha de cerúmen ou sangue.

- Timpanometria, que teve por objetivo identificar alterações 
de orelha média como otites e perfuração timpânica e

- Pesquisa do Nistagmo e Vertigem de Posição nas posições: decúbito dorsal, decúbito lateral direito e esquerdo, decúbito dorsal com a cabeça pendente, decúbito dorsal com a cabeça pendente para direita e para esquerda e sentado. Durante a pesquisa, os sujeitos se encontraram sobre uma maca. Esta prova foi realizada, pois de acordo com Bolsen e Torres ${ }^{15}$, a presença de nistagmo e/ou vertigem em qualquer das posições referidas já indica alteração irritativa, embora não defina a sua localização e com Caovilla e col. ${ }^{16}$ que afirmam que a presença de vertigem e/ou nistagmo de posição é muito freqüente em pacientes com vestibulopatias.

- Avaliação Eletronistagmográfica: Calibração, Nistagmo Espontâneo de olhos abertos e olhos fechados, Nistagmo Semi-Espontâneo para direita e para esquerda, Rastreio Pendular, Nistagmo Optocinético para direita e para esquerda realizados com o paciente sentado em uma cadeira; Recalibração, Nistagmo Pré-Calórico (olhos fechados) e Nistagmo Pós-Calórico (com olhos fechados e com olhos abertos para a pesquisa do EIFO) realizados com o paciente deitado em uma maca na posição I de Brünnings, ou seja, com a cabeça em um ângulo de $30^{\circ}$ do plano horizontal ${ }^{17,18}$. Para a realização da eletronistagmografia, foi feita limpeza da pele dos indivíduos, com álcool, precedendo a colocação dos eletrodos: um no canto externo do olho direito, um no canto externo do olho esquerdo e um na fronte (fio terra). Os sujeitos foram colocados de forma a obter-se uma distância de 1,5 metros da unidade de controle biológico (para realização da calibração e recalibração), fazendo com que o registro da pena inscritora do eletronistagmógrafo fosse ajustado de forma que cada 1 grau de desvio dos olhos correspondesse a 1 milímetro de deslocamento, da pena, no papel milimetrado de inscrição?. Utilizou-se ar para estimulação calórica a $20^{\circ} \mathrm{C}$ (fria) e $42^{\circ} \mathrm{C}$ (quente) com tempo de 80 segundos, na seguinte ordem: fria na orelha esquerda, fria na orelha direita, quente na orelha direita, quente na orelha esquerda ${ }^{17}$. O tempo de registro (olhos fechados) foi de 30 segundos, com início 10 segundos após o término do estímulo calórico, para a observação do período de maiores respostas ${ }^{15}$. Dentro deste período, escolheram-se três nistagmos para serem medidos e desta forma foi calculada a média aritmética das VACLs obtidas?.

Fez-se uso dos seguintes materiais: gel condutor, gaze, álcool, fita adesiva, Unidade de Controle Biológico para Nistagmografia Berger Modelo CB-115 (cruz biológica), Estimulador Optocinético Berger Modelo TB - 113, Vectonistagmógrafo Berger Modelo VN - 316, rolo de papel de registro milimetrado e Otocalorímetro Berger Modelo AR - 314 .

Ao final das avaliações foram realizadas a avaliação qualitativa dos resultados (observação dos traçados) e os cálculos de PDN para nistagmo optocinético; e PDN e PL (quando não estavam presentes arreflexia, hipo ou hiper- reflexia) para nistagmo pós-calórico. Para análise dos resultados, utilizaram-se os valores propostos por GANANÇA e col. ${ }^{17}$ pela especificação à prova calórica à ar tanto para PDN quanto para PL.

\section{RESULTADOS E DISCUSSÃO}

Em 100\% da amostra avaliada, não foi observada a presença isolada de nistagmo de posição. Este dado concorda com Silva e col. ${ }^{14}$ que afirmam que não há presença de nistagmo de posição em indivíduos com alterações metabólicas. Em contrapartida, Biurrun e col. ${ }^{6}$ relataram a presença de nistagmo de posição em 26,1\% dos sujeitos portadores de Diabetes Mellitus tipo I que avaliaram.

$16,7 \%$ dos indivíduos manifestaram presença de vertigem paroxística e fatigável, sem nistagmo, nas posições cabeça pendente para direita e para esquerda.

Ganança e col. ${ }^{17}$ afirmam que a vertigem posicional ou de posicionamento indica distúrbio geralmente do sistema vestibular periférico.

Caovilla e col. ${ }^{16}$, Ganança e col. ${ }^{19}$ e Bolsen e Torres ${ }^{15}$, a presença de vertigem em qualquer posição durante esta prova indica alteração vestibular irritativa sem definição de sua localização.

100\% dos sujeitos avaliados demonstraram traçado regular à calibração. Este dado está de acordo com Bolsen e Torres $^{15}$, que dizem ser o traçado regular (retangular repetitivo), o esperado à calibração do olhar em pacientes normais ou com alterações vestibulares periféricas; com Ganança e col. ${ }^{19}$, que referem alterações principalmente nas crises vertiginosas e nas síndromes vestibulares centrais; com Silva e col. ${ }^{14}$ que dizem que os movimentos sacádicos em indivíduos com labirintopatia de origem metabólica normalmente não apresentam anormalidades; e Almeida ${ }^{20}$, que encontrou calibração regular em 100\% dos sujeitos avaliados com alteração metabólica de origem tireóidea.

Não foi observada presença de nistagmo espontâneo de olhos abertos em 100\% da amostra, o que indicaria normalidade, pois, de acordo com Lopes Filho e Campos ${ }^{21}$, o nistagmo espontâneo de olhos abertos é sempre patológico. Este dado concorda com Silva e col. ${ }^{14}$, que dizem ser o nistagmo espontâneo geralmente sem anormalidades em indivíduos com labirintopatias de origem metabólica; e discorda dos achados de Almeida ${ }^{20}$, que encontrou presença de nistagmo espontâneo de olhos abertos em 11 dos 25 sujeitos avaliados com alteração metabólica de origem tireóidea. Para Bolsen e Torres ${ }^{15}$, nas síndromes periféricas, o nistagmo espontâneo de olhos abertos só se manifesta nas crises labirínticas, o que não ocorreu durante a coleta de dados da presente amostra.

$100 \%$ da amostra não apresentou nistagmo espontâneo. Este dado concorda com Caovilla e col. ${ }^{22}$ e Silva e col. ${ }^{14}$, que dizem que o nistagmo espontâneo geralmente não apresenta anormalidades em indivíduos com labi- 
rintopatias de origem metabólica; e Bittar e col..$^{6}$ que dizem ser a prova calórica o único exame alterado em pacientes diabéticos. O presente achado vai ao encontro dos achados de Almeida $^{2}$, que encontrou presença de nistagmo espontâneo de olhos fechados em $75 \%$ da amostra avaliada com disfunção metabólica tireóidea; e de Biurrun e col. ${ }^{12}$ que, pesquisando as anormalidades eletronistagmográficas em sujeitos portadores de Diabetes Mellitus tipo I, relataram que $15,2 \%$ da amostra apresentou nistagmo espontâneo. Os autores não referiram se o nistagmo ocorreu de olhos abertos, fechados, ou ambos.

Em 100\% da amostra avaliada, não foi observada a presença de nistagmo semi-espontâneo. Nenhum dos sujeitos avaliados estava sob crise labiríntica durante a coleta de dados desta pesquisa, concordando com Lopes Filho e Campos ${ }^{21}$, que dizem que o nistagmo semi-espontâneo de olhos abertos pode surgir apenas durante as crises labirínticas nas síndromes periféricas.

Bittar e col. ${ }^{6}$, Ramos e col. ${ }^{8}$, Biurrun e col. ${ }^{12}$ e Almeida ${ }^{20}$ não citam este teste em suas pesquisas.

$58,3 \%$ da amostra apresentou rastreio pendular do tipo I e 41,7\% dos sujeitos apresentou rastreio pendular do tipo II, o que para Lopes Filho e Campos ${ }^{21}$, Bolsen e Torres ${ }^{15}$ e Mor e col. ${ }^{9}$ caracterizam padrões de normalidade ou síndrome periférica fora dos momentos de crise. Estes dados

Tabela 1.1. Valores de VACL da prova calórica dos 12 sujeitos avaliados

\begin{tabular}{|c|c|c|c|c|}
\hline Sujeitos & $20 \circ \mathrm{OD}$ & 20ㅇ OE & $42^{\circ} \mathrm{OE}$ & $42^{\circ}$ OD \\
\hline 1 & $15,5^{\circ} / \mathrm{s}$ & $12,5^{\circ} / \mathrm{s}$ & $8,75^{\circ} / \mathrm{s}$ & $12,50 / \mathrm{s}$ \\
\hline 2 & $10,5^{\circ} / \mathrm{s}$ & $12,5^{\circ} / \mathrm{s}$ & $70 / \mathrm{s}$ & $9,5 \% / \mathrm{s}$ \\
\hline 3 & $10,5^{\circ} / \mathrm{s}$ & 11ㅇ/s & $90 / \mathrm{s}$ & $13 \% / s$ \\
\hline 4 & $5,50 / \mathrm{s}$ & $12,25 \mathrm{o} / \mathrm{s}$ & $80 / 5$ & $9,5 \% / \mathrm{s}$ \\
\hline 5 & $7,5 \% / \mathrm{s}$ & $12 \mathrm{o} / \mathrm{s}$ & $25 \% / s$ & $70 / \mathrm{s}$ \\
\hline 6 & 5ㅇs & $23,80 / 5$ & $11 \mathrm{o} / \mathrm{s}$ & $11,50 / \mathrm{s}$ \\
\hline 7 & $5,4 \% / \mathrm{s}$ & $8,1 \% / s$ & $4 \% / s$ & $4,19 / \mathrm{s}$ \\
\hline 8 & 6이s & $110 / \mathrm{s}$ & $6 \% / s$ & $12,5^{\circ} / \mathrm{s}$ \\
\hline 9 & $4,5 \% / s$ & $5,5 \% / s$ & $70 / \mathrm{s}$ & 9ㅇs \\
\hline 10 & $10 \% / s$ & 3ㅇ/s & $60 / 5$ & 5,5 ㅇs \\
\hline 11 & $17 \mathrm{o} / \mathrm{s}$ & $10 \% / s$ & 15의s & 11,5 o $/ \mathrm{s}$ \\
\hline 12 & $18 \% / s$ & $13,50 / \mathrm{s}$ & 20,5 o/s & 6,5 ㅇs \\
\hline
\end{tabular}

Tabela 1.2. Dados da prova calórica

\begin{tabular}{cc}
\hline Sujeitos & Valor Final \\
\hline 1 & $13,7 \%$ \\
2 & $11,39 \%$ \\
3 & $10,34 \%$ \\
4 & $23,4 \%$ \\
5 & $44,7 \%$ \\
6 & $37,6 \%$ \\
7 & $12,3 \%$ \\
8 & $23,9 \%$ \\
9 & $11,5 \%$ \\
10 & $30,6 \%$ \\
11 & $19,6 \%$ \\
12 & $44,5 \%$ \\
\hline
\end{tabular}

estão em acordo com Silva e col. ${ }^{14}$ que afirmam que o rastreio pendular não apresenta anormalidades em indivíduos com labirintopatia de origem metabólica e com Almeida ${ }^{20}$, que encontrou rastreios pendulares de tipo I e II nos 25 sujeitos avaliados com disfunção metabólica de origem tireóidea.

$100 \%$ da amostra apresentou simetria à avaliação optocinética. Este dado concorda com a pesquisa de Almeida ${ }^{20}$, que encontrou simetria em 100\% dos pacientes avaliados com disfunção metabólica de origem tireóidea. Silva e col. ${ }^{14}$ também afirmam que a pesquisa do nistagmo optocinético se apresenta geralmente sem anormalidades em sujeitos com labirintopatia de origem metabólica.

Nesta amostra, 100\% não apresentou nistagmo à prova pré-calórica.

Ganança e col. ${ }^{17}$, Bolsen e Torres ${ }^{15}$ e Mor e col. ${ }^{19}$ aconselham a pesquisa do nistagmo pré-calórico de OF antes do início da estimulação térmica para verificar a influência deste nistagmo no resultado da prova calórica.

$100 \%$ apresentou normo-reflexia na análise de VACL em valores absolutos. Para Ganança e col. ${ }^{17}$, a normo-reflexia compreende valores de VACL entre 2 e 28\%/s; para Bolsen e Torres ${ }^{15}$, entre 3 e $36^{\circ} / \mathrm{s}$.

Estes dados discordam de Almeida ${ }^{20}$, que em sua pesquisa com pacientes com alterações metabólicas de origem tireóidea, encontrou hiper-reflexia em 20\% dos sujeitos avaliados; de Aantaa e Lehtonen ${ }^{23}$, que relataram o predomínio de hipo-reflexia em indivíduos diabéticos; de Ramos e col. ${ }^{8}$ que relataram incidência elevada de hiperreflexia labiríntica em sujeitos com queixa de distúrbio corporal e/ou da audição associada à insulinemia; de Cojazzi ${ }^{24}$ apud Biurrun e col. ${ }^{12}$, que referiu como o achado principal a hiper-reflexia; e de Biurrun e col. ${ }^{12}$, que encontrou hiporeflexia em 10 dos 46 sujeitos avaliados com Diabetes Mellitus tipo I.

$41,6 \%$ apresentaram resultados normais e $58,4 \%$ apresentaram alteração à prova calórica, conforme o Gráfico.

Estes dados vão ao encontro dos achados de Silva e col. ${ }^{14}$ que dizem que na prova calórica com ar, uma das alterações encontradas em indivíduos com labirintopatia de origem metabólica é o Predomínio Direcional do Nistagmo, demonstrando um quadro de comprometimento irritativo; de Caovilla e col..$^{22}$ que encontraram como um dos achados predominantes o Predomínio Direcional do Nistagmo; e de Biurrun e col. ${ }^{12}$, que relataram a presença de Predomínio Direcional do Nistagmo em portadores de Diabetes Mellitus. Os dados desta pesquisa discordam de Camiasca ${ }^{25}$ apud Biurrun e col. ${ }^{12}$ que encontrou Predomínio Labiríntico em 45\% e Predomínio Direcional do Nistagmo em 18,7\% dos indivíduos avaliados com Diabetes Mellitus tipo I; de Cojazzi ${ }^{24}$ apud Biurrun e col. ${ }^{12}$, que referiu somente alguns casos de preponderância direcional do nistagmo em suas pesquisas; de Bittar e col. ${ }^{6}$, que dizem que, em alguns casos, os exames eletronistagmográficos podem apresentarem-se normais em pacientes diabéticos. 


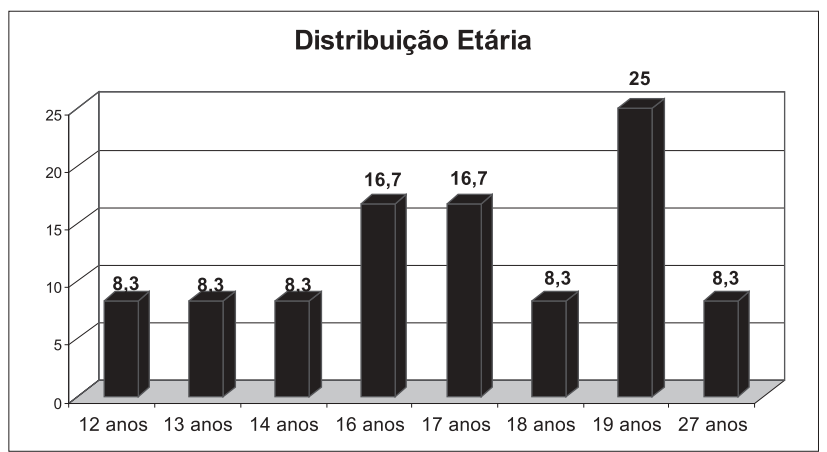

Gráfico 1.1 Distribuição Etária

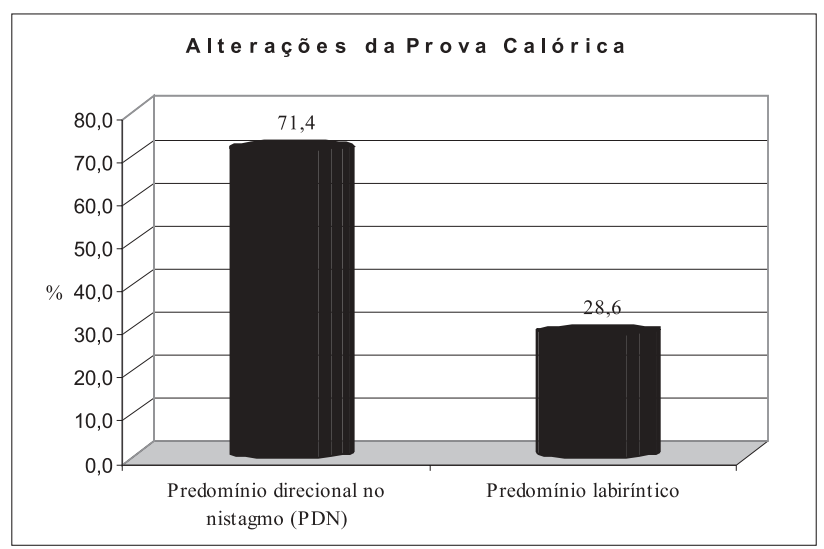

Gráfico 2.2 Alteração da Prova Calórica

Dentre os sujeitos da amostra, 33,33\% tiveram resultados de acordo com os padrões de normalidade, enquanto que $50 \%$ tiveram como resultado síndrome vestibular periférica irritativa sem definição de sua localização e 16,7\% síndrome vestibular periférica deficitária.

Dos 8 (100\%) exames alterados, 75\% tiveram como resultado final Síndrome Vestibular Periférica Irritativa (por PDN e Vertigem de posição) e 25\% Síndrome Vestibular Periférica Deficitária (PL).

Os resultados encontrados, indicando $75 \%$ da amostra com Síndrome Vestibular Irritativa é, segundo Gil ${ }^{26}$, descritivamente relevante, e confirmam o que refere Silva e col. ${ }^{14}$ ao afirmar que a avaliação otoneurológica em sujeitos com labirintopatia de origem metabólica apontam para um padrão de comprometimento irritativo e que as labirintopatias de origem metabólica se caracterizam por levar o portador da alteração à Síndrome Vestibular Periférica; de Ramos e col. ${ }^{8}$ que salientaram a incidência elevada de hiper-reflexia labiríntica à prova calórica em sujeitos examinados com queixa de distúrbio corporal e/ou da audição associada à insulinemia; de Cojazzi ${ }^{24}$ apud Biurrun e col. ${ }^{12}$, que referiu como o achado principal a hiper-reflexia bilateral; e discordam de Aantaa e Lehtonen ${ }^{23}$ que relatam ter encontrado predominantemente reações calóricas diminuídas em indivíduos diabéticos.

$87,5 \%$ apresentaram respostas alteradas à avaliação eletronistagmográfica e em somente $12,5 \%$ dos sujeitos, o diagnóstico foi definido a partir da pesquisa da vertigem de posição.

Mesmo não tendo como objetivo de estudo neste trabalho, entre 100\% dos indivíduos avaliados, 25\% manifestou queixa de tontura em situações específicas de hipoglicemia, enquanto que $75 \%$ não citou sua presença, conforme demonstra o Gráfico. 100\% dos sujeitos que manifestaram este sintoma tiveram como resultado final à eletronistagmografia síndrome vestibular irritativa. Dentre os $100 \%$ dos exames alterados, $62,5 \%$ não referiram tontura.

Esses dados concordam com Jerger e Jerger ${ }^{27}$, pois os autores acreditam que aproximadamente $20 \%$ dos pacientes diabéticos podem queixar-se de tontura; com Olefsky e col. ${ }^{28}$, Leher e col. ${ }^{29}$ e Perez e col. ${ }^{11}$ que acreditam numa possível conexão entre dieta dos pacientes e a queixa de vertigem. Todos sujeitos da amostra mantêm um acompanhamento nutricional; com Bittar e col. ${ }^{6}$, Ramos e col. ${ }^{8}$ e Ferreira e col. ${ }^{7}$ dizem que, pelo fato da OI não ter reserva energética armazenada, pequenas variações de glicose já são capazes de influenciar em seu funcionamento; com Ferreira Jr. e col. ${ }^{7}$ que relacionaram a redução de episódios vertiginosos à orientação dietética e exercícios físicos; com Ramos e col. ${ }^{8}$ que dizem que o diabético que faz uso regular de insulina tem tonturas com menos freqüência, fato que explicaria a pequena incidência de queixa de tontura na presente amostra, pois se tratam de portadores de DM Tipo I que fazem uso regular de insulina; com Silva e col. ${ }^{8}$ que afirmam que a maioria dos casos de alteração da OI em indivíduos portadores de alterações metabólicas ocorre em função da hipoglicemia; com Ferreira Jr. e col. ${ }^{7}$ que encontraram número significativo de indivíduos com disfunção metabólica associada a episódios vertiginosos e hipoglicêmicos; e com Perez e col. ${ }^{11}$ que consideram que no DM, a alteração seria simétrica, o que causaria sintomas clínicos somente em casos de considerada severidade; e discordam de Ramos e col. ${ }^{8} \mathrm{e}$ Lopes Filho e Campos ${ }^{21}$ que afirmam que o DM pode estar associado ou não a sintomas como zumbido, vertigem e tontura.

O efeito do DM deve ser avaliado como as demais complicações diabéticas usuais, pois, com o diagnóstico etiológico, pode ser possível o auxílio na prevenção das complicações desta doença.

Sugere-se a realização de um estudo longitudinal, com uma amostra maior desta população, a fim de comprovar o comprometimento, de causa metabólica, nas alterações vestibulares encontradas.

\section{CONCLUSÃO}

Por meio dos resultados coletados, verificou-se, no grupo pesquisado, alteração vestibular. 75\% dos resultados 
alterados apresentaram presença de comprometimento vestibular irritativo sem definição de sua localização e, portanto, observou-se que o órgão vestibular deva ser incluído na lista de órgãos e tecidos afetados pelo DM.

\section{AGRADECIMENTOS}

Agradeço à Associação Riograndense de Apoio ao Diabético (ARAD) que se mostrou disponível a auxiliar na concretização desta pesquisa; aos voluntários participantes da amostra deste estudo, pois sem a colaboração dos mesmos, o trabalho seria inviável; às colegas Geneci Keller, Gislaine Krause, Raquel Hendges, Tatiane Hackenhaar e Verônica Borges que se dispuseram a ajudar na realização da coleta de dados; à Fga. Cíntia de Paula, que sempre esteve presente nos momentos em que se fez necessário; e à minha querida amiga Gisele Lins, que soube me apoiar em momentos cruciais na realização da pesquisa.

Agradeço ao apoio fornecido pela Fga. Elisea Meurer, que me acompanhou e apostou em minhas potencialidades, tendo contribuído no meu desenvolvimento durante a formação acadêmica; e à Fga. Marilda Lobo, por ter dividido comigo uma parcela de seus conhecimentos que, com certeza, servirão de bases fundamentais no início desta caminhada profissional.

\section{REFERÊNCIAS BIBLIOGRÁFICAS}

1. Bevilacqua F, Bensoussan E, Silva JMJ, Castro FS, Carvalhes LP. Manual de Fisiopatologia Clínica. Rio de Janeiro: Atheneu, 1974.

2. Wajchenberg BL et al. Diabetes Melito Insulino-Independente (Tipo I): diagnóstico, etiopatogenia e fisiopatologia. In: Wajchenberg BL. Tratado de Endocrinologia Clínica. São Paulo: Roca, 1992.

3. Oliveira JEP de. http://www.diabetes.org.br/Diabetes, abr 2001

4. Wajchenberg BL. Diabetes Mellitus. São Paulo: v. III Ed. Sarvier, 1970

5. Sociedade Brasileira De Diabetes. http://www.diabetes.org.br/ Diabetes/diabet_set.html acessado em abril, 2001.

6. Bittar RSM, Sanchez TG, Santoro PP, Medeiros IRT de. O metabolismo da Glicose e o Ouvido Interno. São Paulo: @rquivos da Fundação Otorrinolaringologia. v.2, jan/fev/mar, 1988.

7. Ferreira Jr. CA, Guimarães RES, Becker HMG, Silva CDL, Gonçalves TML, Crosara PFTB, Morais MPA de. Avaliação Metabólica do Paciente com Labirintopatia. Belo Horizonte, MG: @rquivos da Fundação Otorrinolaringologia Jan 2000;4:28-32.

8. Ramos RF, Ramos S, Ganança MM, Albernaz PLM, Caovilla HH Avaliação Otoneurológica em Pacientes com Labirintopatias e Alterações da Insulinemia. São Paulo: Acta Who 1989 Mai/ Ago;8(2):63-6.

9. Mor R, Fragoso M, Taguchi CK, Figueiredo JF. Vestibulometria \& Fonoaudiologia: Como realizar e Interpretar. São Paulo: Lovise; 2001. p.186

10. Fangchao MD. Diabetes and hearing impairment in Mexican American Adults: a population-based study. The Journal of Laryngology and Otology 1998;112:835-9.
11. Perez R, Ziv E, Freeman S, Sichel J, Sohmer H. Vestibular EndOrgan Impairment in na Animal Model of Type 2 Diabetes Mellitus. Laryngoscope 2001 Jan;111:110-3.

12. Biurrun O, Ferrer JP, Lorente J, De España R, Gomis R, Trassera J. Asymptomatic Electronystagmographic Abnormalities in Patientes with Type I Diabetes Mellitus. Journal for Oto-Rhino-Laryngology and Its Borderlands 1991;53:335-8.

13. Caovilla HH, Ganança MM, Munhoz MSL, Serafini FF. Síndromes Cocleovestibulares e Hiperinsulinemismo. RBM-ORL Set 1994; 1(2), São Paulo.

14. Silva MLGDa, Munhoz MSLei, Ganança M, Caovilla HH. Quadros Clínicos Otoneurológicos Mais Comuns. Série Otoneurológica. São Paulo, Rio de Janeiro, Belo Horizonte: v. III Ed. Atheneu, 2000.

15. Bolsen YAE, Torres MLB. Interpretando a eletronistagmografia e vectonistagmografia na avaliação vestibular. In: Gama, Márcia Regina (Org). Resolvendo Casos em Audiologia. São Paulo: Plexus; 2001.

16. Caovilla HH, Ganança MM, Munhoz MSL, Silva MLG da Equilibriometria Clínica. São Paulo: Atheneu, 1999. 158p. vol.1.

17. Ganança MM, Caovilla HH, Munhoz MSL, Silva MLGDa; Frazza MM. Etapas da equilibriometria. In: Caovilla et al. Equilibriometria Clínica. São Paulo: Atheneu; 1999. p. 74-8.

18. Garcia FV, Entrudo A, Ferreira AD, Monteiro MC. O Exame Vestibular Convencional e Seus Limites. Estoril: Rapport do II Simpósio Internacional da Vertigem. Organização do GEV Portugal, 1996.

19. Ganança MM, Munhoz MSL, Caovilla HH, Silva MLG da. Utilidade Clínica do Exame Otoneurológico. RBM-ORL Abr 2000;57, São Paulo.

20. Almeida FS de. Disfunção Metabólica Tireóidea e Otoneurologia. Revista Brasileira de Otorrinolaringologia; jul/ago, 1998. Rio de Janeiro: $n^{-}$64:351-8.

21. Lopes Filho O \& Campos CAH de. Tratado de Otorrinolaringologia. São Paulo: Roca, 1994.

22. Caovilla HH, Ganança MM, Munhoz MSL, Silva MLG Da, Frazza MM. O Equilíbrio Corporal e Seus Distúrbios. RBM-ORL Mai/jun 1998;5(3):89-91, São Paulo.

23. Aantaa E, Lehtonen A. Electronystagmografic findings in insulindependent diabetics. Acta Otolaryngol (Stockh) 1981:91:15-18.

24. Cojazzi L. Le Alterazioni Vestibolare nel Diabete. Arscip S Anna Ferrara 1950;3:76-97 apud Biurrun O, Ferrer JP, Lorente J, De España R, Gomis R, Trassera J. Asymptomatic Electronystagmographic Abnormalities in Patientes with Type I Diabetes Mellitus. Journal for Oto-Rhino-Laryngology and Its Borderlands 1991;53:335-8.

25. Camiasca L. L'esame Dell'apparato Cochleovestibulare nel Diabete Mellito. S SCI Med; 5:45-49, 1950 apud Biurrun O, Ferrer JP, Lorente J, De España R, Gomis R, Trassera J. Asymptomatic Electronystagmographic Abnormalities in Patientes with Type I Diabetes Mellitus. Journal for Oto-Rhino-Laryngology and Its Borderlands 1991;53:335-8.

26. Gil AC. Métodos e técnicas de pesquisa social. 3ạ. ed. São Paulo: Atlas, 1999. 206p.

27. Jerger $S$ \& Jerger J. Alterações Auditivas: Um Manual para Avaliação Clínica. SP-RJ-BH: Atheneu, 1989.

28. Olefsky JM, Farquhar JW, Reaven GM. Reappraisal of the role of insulin in hypertriglyceridemia. American Journal of Medicine 1974:551-560.

29. Lehrer JF, Poole DC, Seaman M, Restivo D, Hartman K. Identification and Treatment of Metabolic Abnormalities in Patients With Vertigo. Archives of Internal Medicine 1986 August; 146(8):1497-500. American Medical Association. 